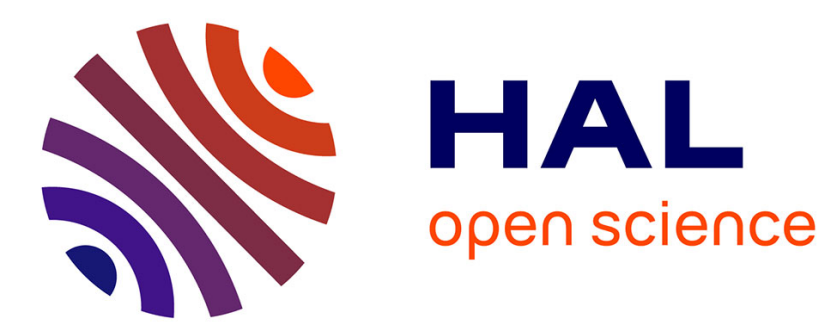

\title{
The linear buncher of SPIRAL beam test of a prototype
}

\author{
A. Chabert, C. Ricaud, L. Boy, B. Monsanglant, W. Le Coz
}

\section{To cite this version:}

A. Chabert, C. Ricaud, L. Boy, B. Monsanglant, W. Le Coz. The linear buncher of SPIRAL beam test of a prototype. Nuclear Instruments and Methods in Physics Research Section A: Accelerators, Spectrometers, Detectors and Associated Equipment, 1999, 423, pp.7-15. 10.1016/S0168-9002(98)01224-8 . in2p3-00005420

\section{HAL Id: in2p3-00005420 \\ https://hal.in2p3.fr/in2p3-00005420}

Submitted on 18 Oct 2017

HAL is a multi-disciplinary open access archive for the deposit and dissemination of scientific research documents, whether they are published or not. The documents may come from teaching and research institutions in France or abroad, or from public or private research centers.
L'archive ouverte pluridisciplinaire HAL, est destinée au dépôt et à la diffusion de documents scientifiques de niveau recherche, publiés ou non, émanant des établissements d'enseignement et de recherche français ou étrangers, des laboratoires publics ou privés. 


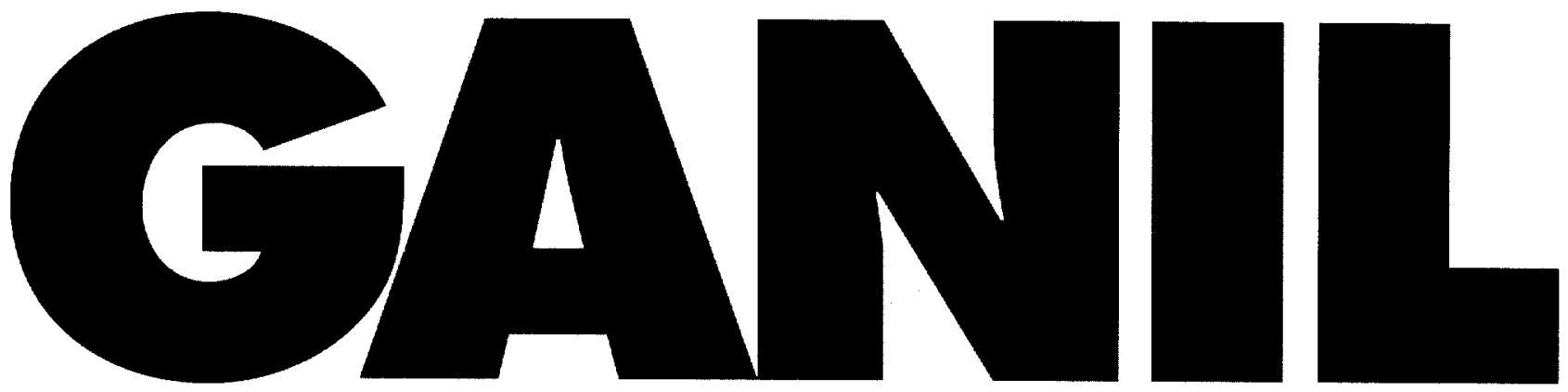

GRAND ACCELERATEUR NATIONAL D'IONS LOURDS - CAEN LABORATOIRE COMMUN IN2P3 (CNRS) - DSM (CEA)

The Linear Buncher of SPIRAL.

Beam test of a prototype.

A.Chabert - Ch.Ricaud - L.Boy

GANIL - Caen.

B.Montsanglant - W.Le Coz.

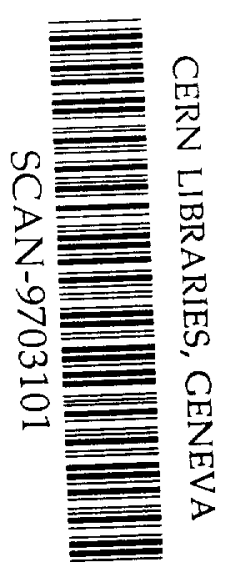

I.P.N - Orsay.

Soumis à NIM

GANIL S 9701

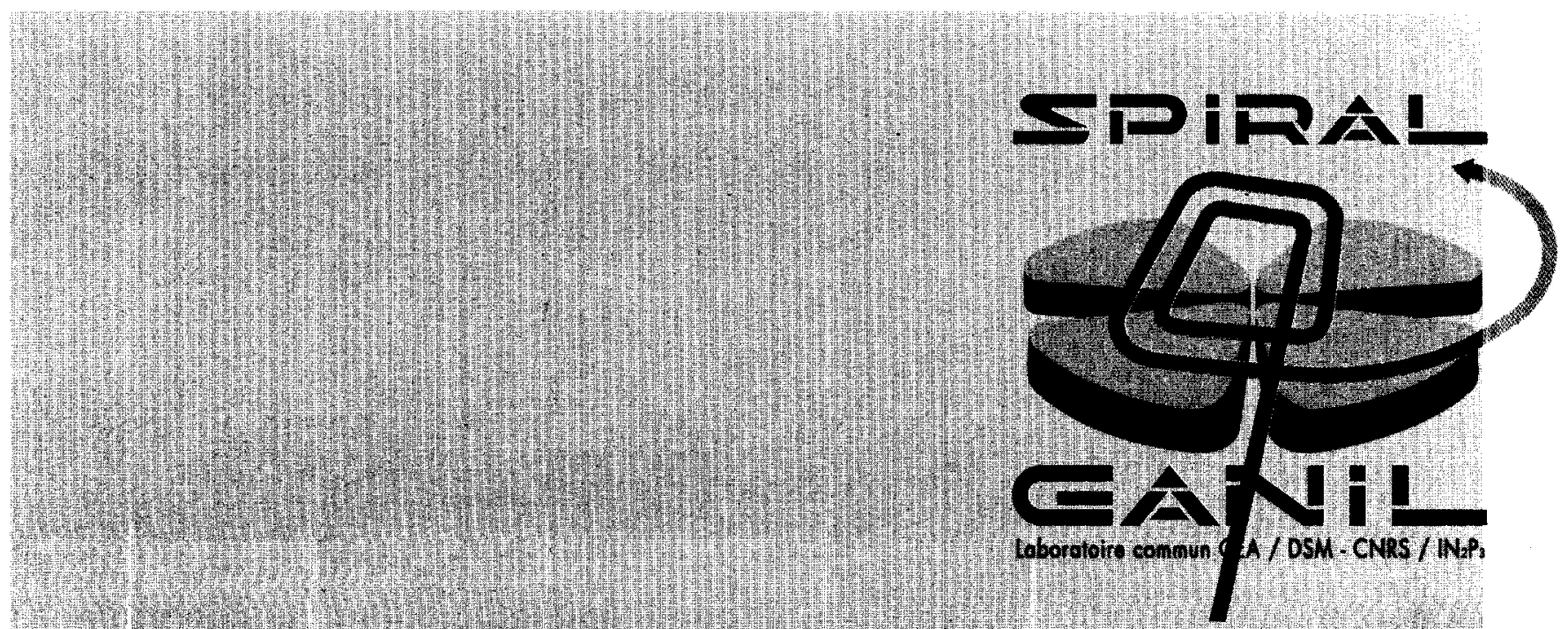




\section{The Linear Buncher of SPIRAL. \\ Beam test of a prototype. \\ A.Chabert - Ch.Ricaud - L.Boy \\ GANIL - Caen. \\ B.Montsanglant - W.Le Coz. \\ I.P.N - Orsay.}

1 - Introduction.

The radioactive ion beam ( RIB ) facility under construction at GANIL ( SPIRAL [1]) will post-accelerate, using a $1.5 \mathrm{~m}$ compact cyclotron ( CIME ) the RIB produced at low energy by the ISOL method. In CIME, the RF frequency ranging from 9.6 to $14.5 \mathrm{MHz}$, we will use 4 harmonics $(\mathrm{h}=2,3,4$ and 5 ) depending on the energy we want, from $\sim 1.7$ to $25 \mathrm{MeV} / \mathrm{A}$.

The RIB are extracted at a maximum voltage of $\sim 34 \mathrm{KV}$ from the ECR ion source so that the wave lenght ( $\beta \lambda$ ) of the beam to be injected into CIME is very low : respectively $\sim 10.68,7.12,7.07$ and $5.65 \mathrm{~cm}$ for $\mathrm{h}=2,3,4$ and 5 . On the other hand the emittance is large : up to $80 \pi \mathrm{mm} . \mathrm{mrad}$.

The overall transmission of the facility has to be as high as possible for these very rare and expensive RIB and then, it was essential to pay a special attention to the bunching system located between the ECR ion source and the cyclotron : a linear single gridded gap buncher has been studied and a prototype has been built and tested on the beam injection line of the GANIL injector C01 having characteristics similar to the one of CIME.

\section{2 - The choice of the buncher for SPIRAL.}

2-1.Generating a linear voltage : being concerned by low energies (low voltages ) and low frequencies, the linear voltage will most often be electronicaly generated. The usual proposed ways are :

it is so simple!

-a pure sinus wave : it gives a rather low bunching efficiency but

-a double drift system [2] which is equivalent to the superposition of several harmonics for approximating a linear voltage : this is the today solution we use in the beam injection line of the GANIL C01. It implies two sets of electrodes and in principle the geometry should be fitted for each $\beta \lambda$. On the other hand the tuning is not so simple as the relative phase and amplitude of the two componants have to be carefully controled.

-the direct sommation of 4 harmonics as proposed and used at Argonne [3] : this is an efficient method but it seems restricted to rather high frequencies and narrow frequency ranges as resonant lines are used which have to 
be tuned according to the frequency. In the same way as above the phases and amplitudes of the 4 harmonics have to be precisely controled.

-the amplification, using a wide band amplifier, of a linear voltage produced at low level as proposed at Texas [4].

-the direct production at the required level of a linear voltage by charging a capacity which is nothing else than the buncher itself. This very elegant method has been proposed at Lanzhou [5] : this is the one we have choosen.

2-2.The buncher electrode system. Two main systems are proposed :

-the drift tube system : it is well suited to "high" values of $\beta \lambda$ and rather low emittances due to the transit time factor effect which implies a small drift tube apperture as compared to its length. A drift tube system is of course fitted to a given $\beta \lambda$, at least if we use a $\beta \lambda / 2$ structure including a drift tube in between two gaps.

-the single gridded gap system which is the choice we are left with at our very low $\beta \lambda$ and large emittances.

\section{2-3.The single gridded gap buncher.}

A sketch of this buncher is shown on the figure $1:$ the positive and increasing linear RF voltage ( we will see in the $\S 4$ how it is produced ) is directly applied to the first electrode, the second one being grounded. The result is a fairly uniform positive high RF field in between the two gridded electrodes ( the effects of the grids have been carefully studied at Argonne [3] ) and unfortunately a stray RF field of low level but of large extent in front of the first electrode.

This stray RF field has to be controled for minimising its effects on the beam, it is shaped by a careful design of the $R F$ electrode and, as will be seen in $\S 3$, by adding a movable grounded plate in front, its position depending on $\beta \lambda$.

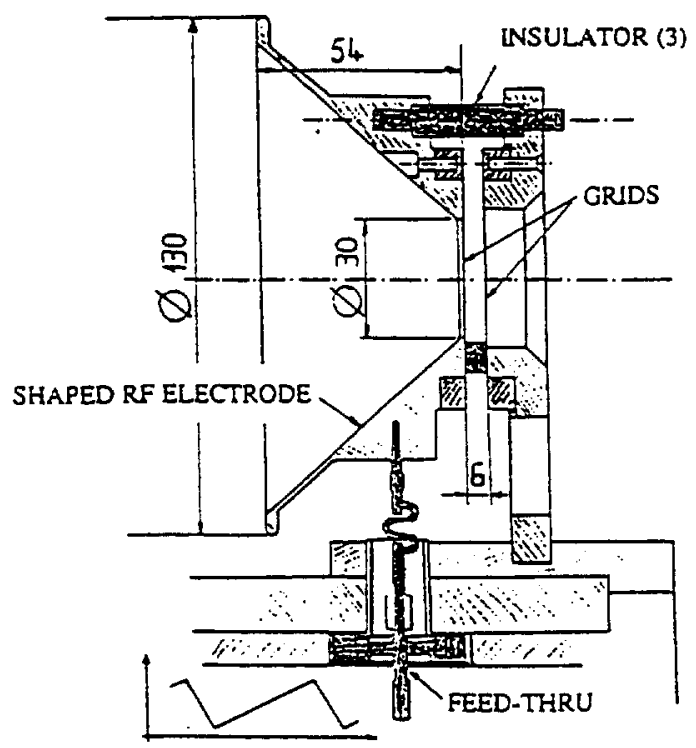

Figure 1 : the electrode geometry of the prototype. 
3 - Simulation of the particle behaviour in the buncher.

3-1.The shape of the RF electrode : for a given shape of the electrode system, including the grounded electrode, the potential is determined using the commercial code PLTMG. The final configuration results from iterations taking into account the results of the particule dynamic simulation inside the successive field patterns.

On the figure 2, we display the shape of the electrodes we have retained for the final design and the resulting stray fields on the axis for two extreme positions of the grounded plate.
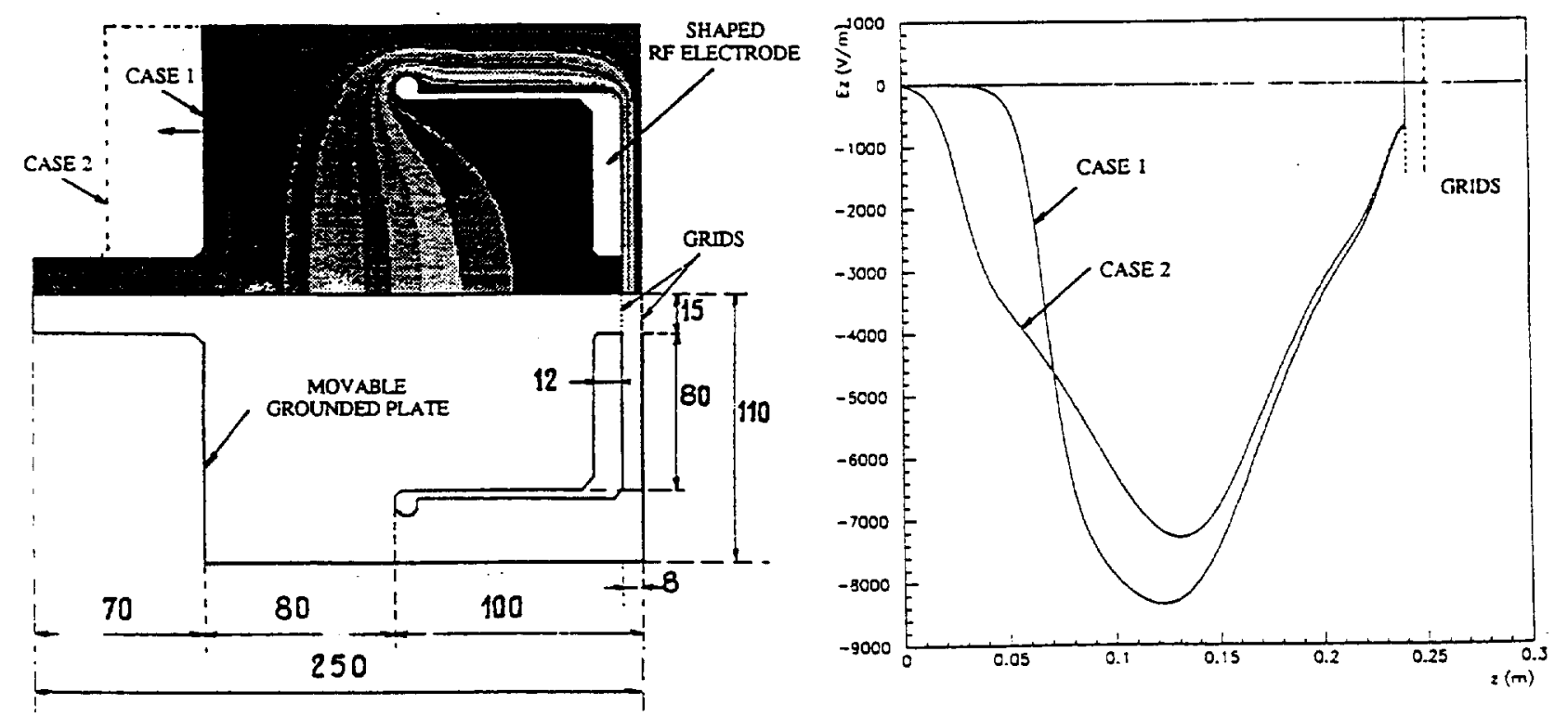

Figure 2 : the electrodes of the final design and the stray fields on the axis.

3-2.Particle dynamics : results of the simulation in the final design.

To perform the simulations we have used our multiparticle code SOSO, the $\mathrm{dc}$ initial beam being represented by 2001 test ions uniformely distributed in the 5 dimension phase space $\left(x, p_{x}, y, p_{y}, \delta w / w\right)$ and independently over $360^{\circ}$.in phase. The field outside of the axis is calculated to the first order from the field on the axis. The time variation of the applied voltage is either a pure sawtooth (75\% ramping time) or the one measured on the buncher prototype ( see $\S 4$ ).

The results are given, downstream of the buncher, after a $7.5 \mathrm{~m}$ drift space without any focusing element. The saw-tooth amplitude is adjusted for obtaining a phase focus at this position.

On the figure 3 we display, for a given case (given $\beta \lambda$ and geometry, pure sawtooth voltage), the energy history along the buncher of 3 on-axis particles with initial $\delta \mathrm{w} / \mathrm{w}=0$ : the central one and two others located $\pm 120^{\circ}$ appart. For the central particle, the energy gain in the stray field is of course almost completely 
cancelled after the gap crossing and, due to the choice of the geometry for this given $\beta \lambda$, the energy dispersion of the two other particles ( $\pm 120^{\circ}$ out of phase ) is almost symetrical and so they will be bunched in the same way.

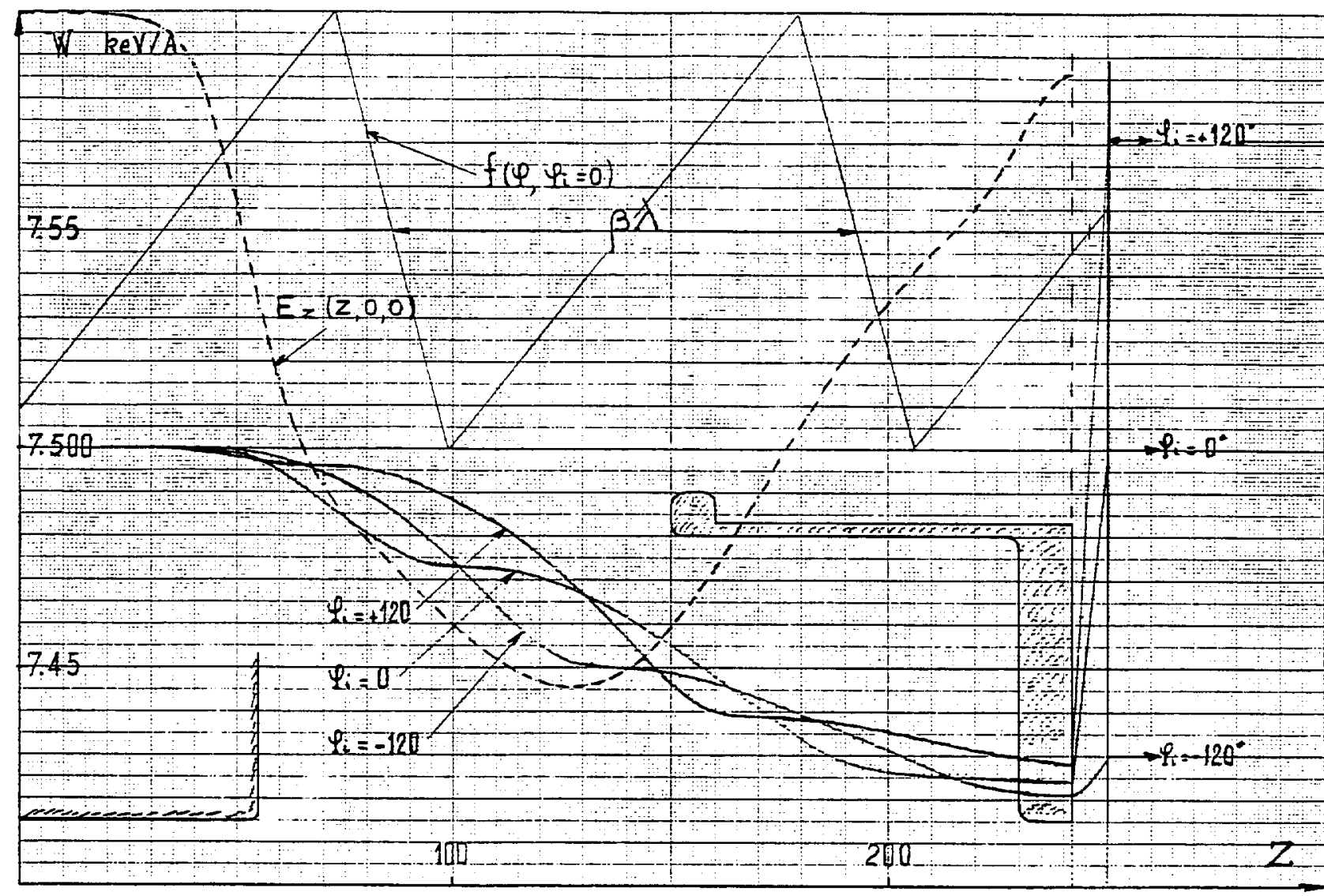

Figure 3

On the figure 4 we display, for a given $\beta \lambda$, the same amplitude of a pure sawtooth voltage and 3 positions of the grounded plate the bunched part of the beam at the phase focus. The saw-tooth amplitude has been optimised for the $d=0$ position. We see that the movable element allows for a rather fine control of the beam linearity over the whole linear phase range of the applied voltage.
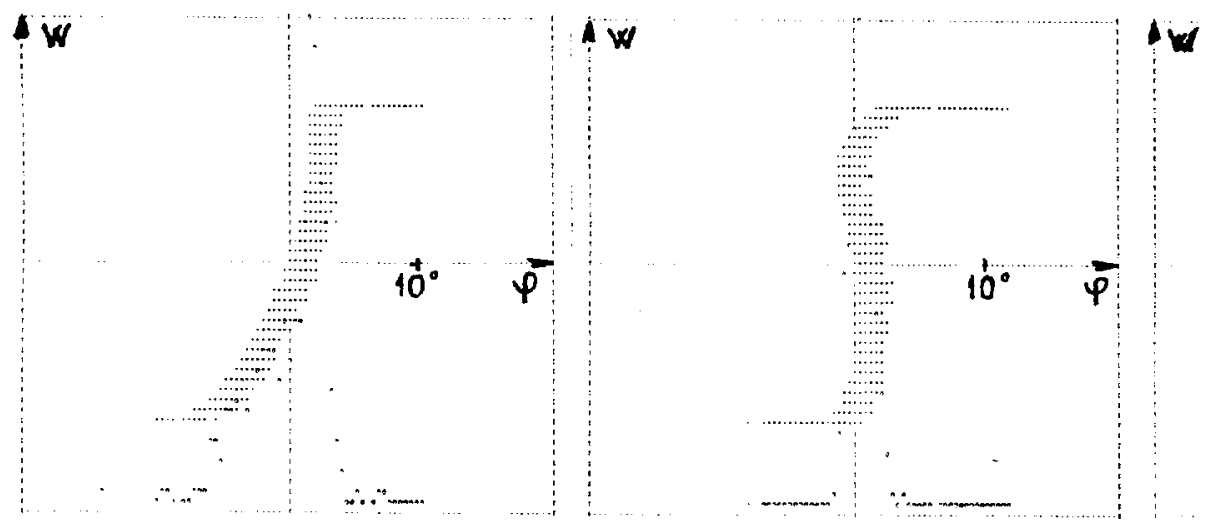

$\mathrm{d}=-20 \mathrm{~mm}$

$$
\mathrm{d}=0
$$$$
\mathrm{d}=20 \mathrm{~mm}(\text { case } 1)
$$

Figure $4:{ }_{20} \mathrm{Ne}^{+5}, \mathrm{~V}_{\mathrm{ECR}}=30 \mathrm{KV}, \beta \lambda=10.68 \mathrm{~cm}, \mathrm{~V}_{\text {buncher }}=602 \mathrm{~V}$.

Transverse emittance : $40 \pi \mathrm{mm} \cdot \mathrm{mrad}$ in each plane. $\Delta \mathrm{w} / \mathrm{w}=0$. 
On the figure 5 we display, in the case of a pure sawtooth voltage, the bunched beams obtained for 3 harmonics we will use in SPIRAL( $h=2,3,5$. The case $h=4$ having almost the same $\beta \lambda$ as $h=3$ is not displayed ). The movable plate position and the sawtooth amplitude have been optimised in each case keeping the same gridded electrodes. We clearly see the emittance effect which widens the distribution of the bunched beam as $\beta \lambda$ decreases.

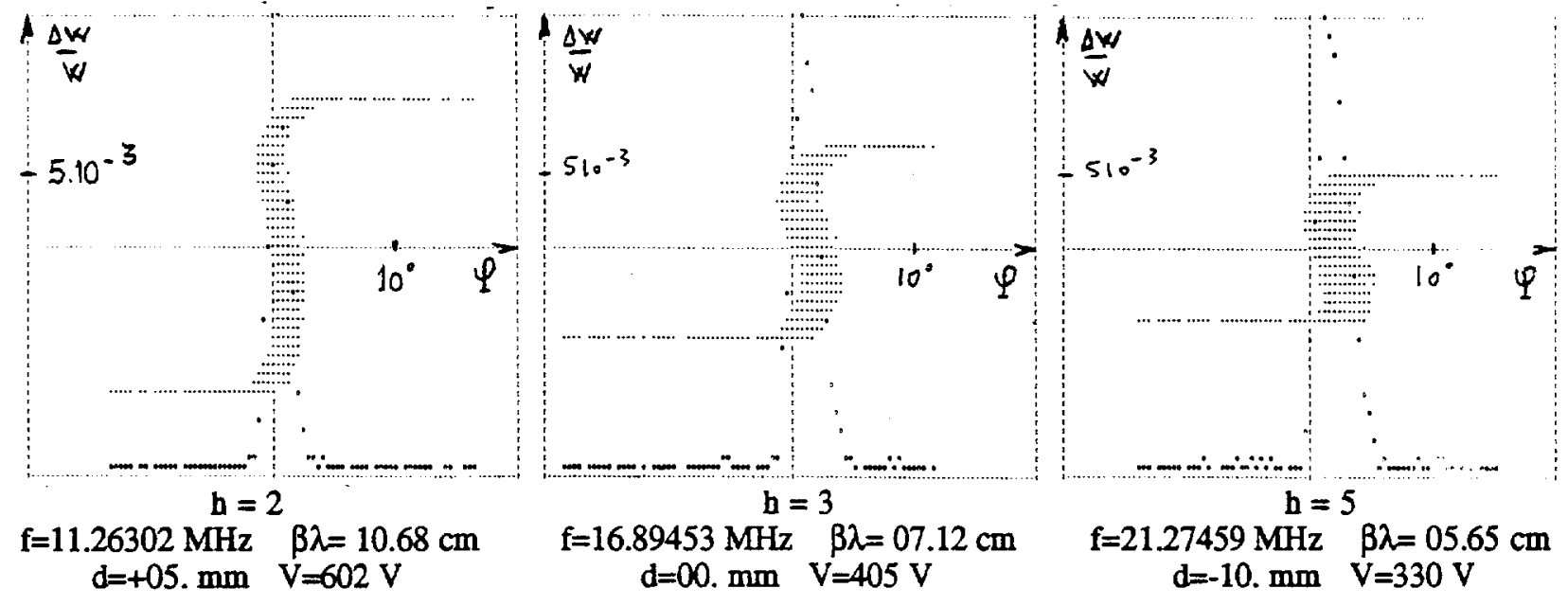

Figure $5:{ }_{20} \mathrm{Ne}^{+5}, \mathrm{~V}_{\mathrm{ECR}}=30 \mathrm{KV}$.

Transverse emittance : $40 \pi \mathrm{mm} \cdot \mathrm{mrad}$ in each plane. $\Delta \mathrm{w} / \mathrm{w}=0$.

On the figure 6 we compare the results obtained using either the pure sawtooth voltage or the one measured on the prototype ( see $\S 4$ ). The amplitude of the sawtooth and its linear, or almost linear part ( $86.6 \%$ ) are the same in the two cases. We can see the great importance to obtain as linear as possible the "sawtooth" voltage.

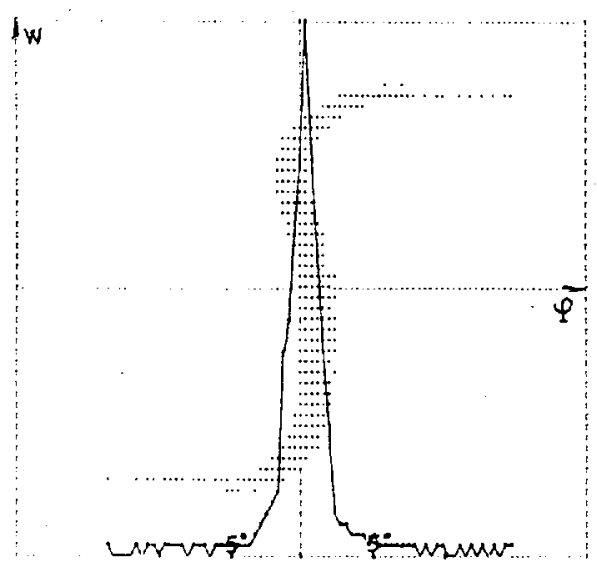

saw-tooth voltage

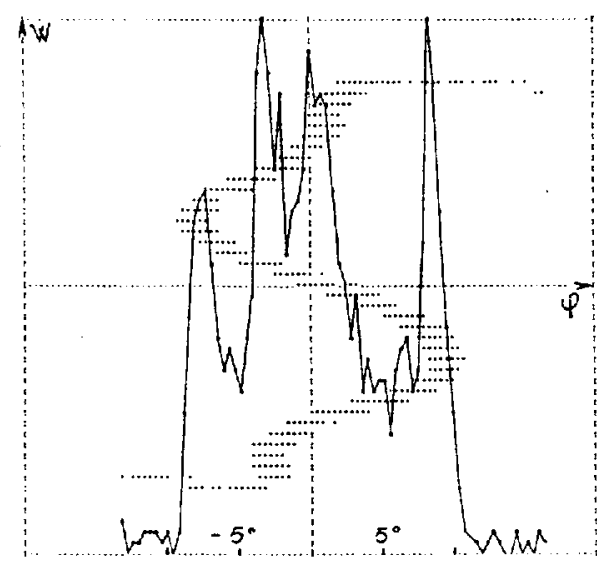

prototype voltage

Figure $6:{ }_{20} \mathrm{Ne}^{+5}, \mathrm{~V}_{\mathrm{ECR}}=30 \mathrm{KV}, \beta \lambda=10.68 \mathrm{~cm}, \mathrm{~V}_{\text {buncher }}=602 \mathrm{~V}$.

Transverse emittance : $40 \pi \mathrm{mm}$.mrad in each plane. $\Delta \mathrm{w} / \mathrm{w}=0$.

We have also checked that a possible biasing of the saw-tooth voltage does not result in any difference on the bunched beam characteristics.

The theoretical study and the results of the simulations being quite encouraging, we have decided to built a prototype and to test it on a real beam line. 
4 - The high voltage saw-tooth generator.

4-1. Principle : in order to avoid the distorsion introduced by a wide band amplifier, we choose a direct high voltage sawtooth generator as proposed at Lanzhou [5].

To do that, we charge a capacity, the buncher electrode, through an inductance, by a constant current. A tube acts as a fast switch and discharge this capacity very rapidely under the control of an input pulse. A capacitive probe allows for the visualisation of the sawtooth. The bloc diagram of this generator is shown on the figure 7 .

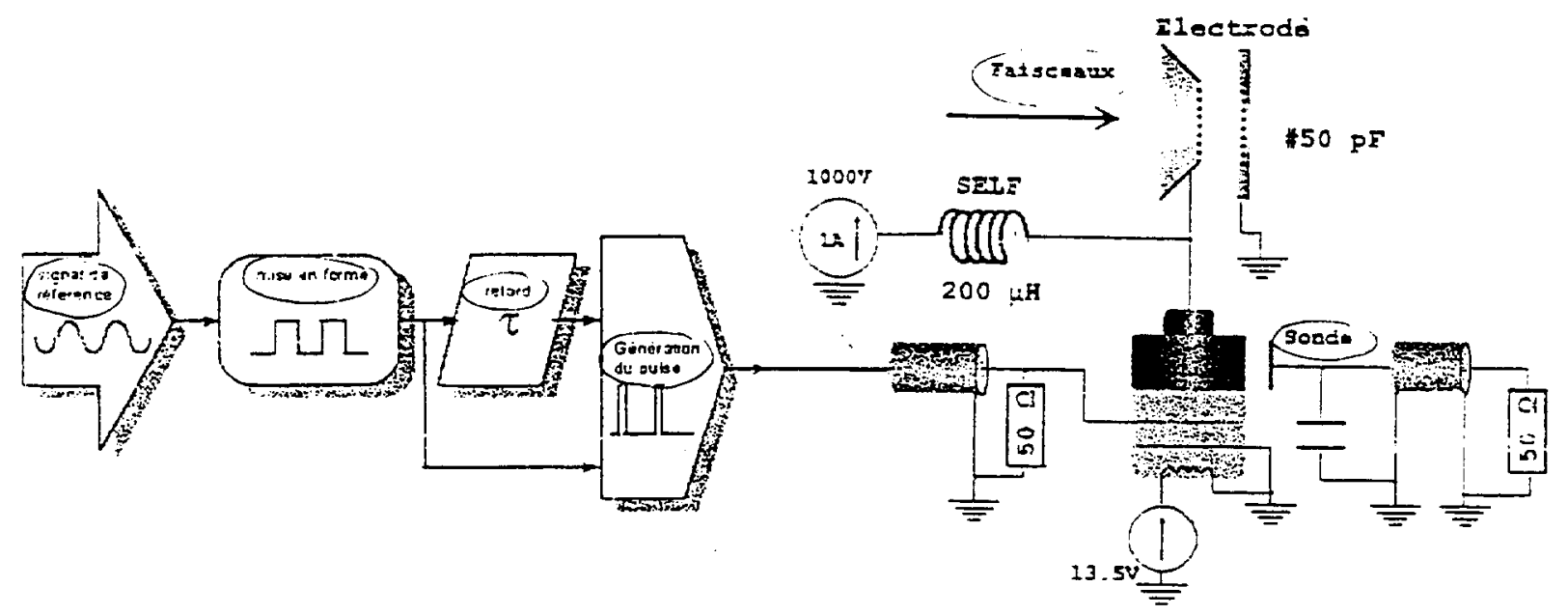

Figure 7 : the sawtooth generator.

4-2.The control pulse : a reference sinus wave ( 5 to $15 \mathrm{MHz}$ in our case ) is used to obtain two delayed rectangular pulses which are combined to give a short one having a width controled by the delay between the first two pulses (in our case this width is fixed and equal to $20 \mathrm{~ns}$ ). This short pulse is then amplified and applied on the grid of the tube.

Entrance capacity and fast commutation are the important considerations for this stage. We use a DMOS technology which gives very short transition times and allows to obtain, in pulsed mode, the high potentials and currents required to drive the tube from a distant location.

4-3.The high voltage signal : the sawtooth amplitude is controled by a high voltage power supply ( $1000 \mathrm{~V}-2 \mathrm{~A}$ in our case ). This voltage is applied on the tube anode through an inductance which have to be very carrefuly designed in order to obtain a good linearity of the sawtooth.

The tube is the EIMAC 3CPX800A7 triode (power up to $800 \mathrm{~W}$, large bandwidth and small output capacity well suited to a pulsed mode ). This tube present the advantage to have a 0 cut-off voltage thus avoiding the use of any polarisation

4-4.The sawtooth signal : we use a capacitive probe around the anode to display the signal. The result is shown on the figure 8 . The linear part of the sawtooth of this prototype generator (always positive and increasing ) presents a small oscillation ( $<4 \%$ over the linear part $\sim 86 \%$ of the period at $7 \mathrm{MHz}$ ) which 
comes from the inductance design which will have to be refined in the final design. The effects of this oscillation can be seen on the figure 6 .

In the same time we will try to reduce the buncher capacity by a carreful design of the electrodes and a better optimisation of the grids thus improving the rapidity of the discharge and the transparency.

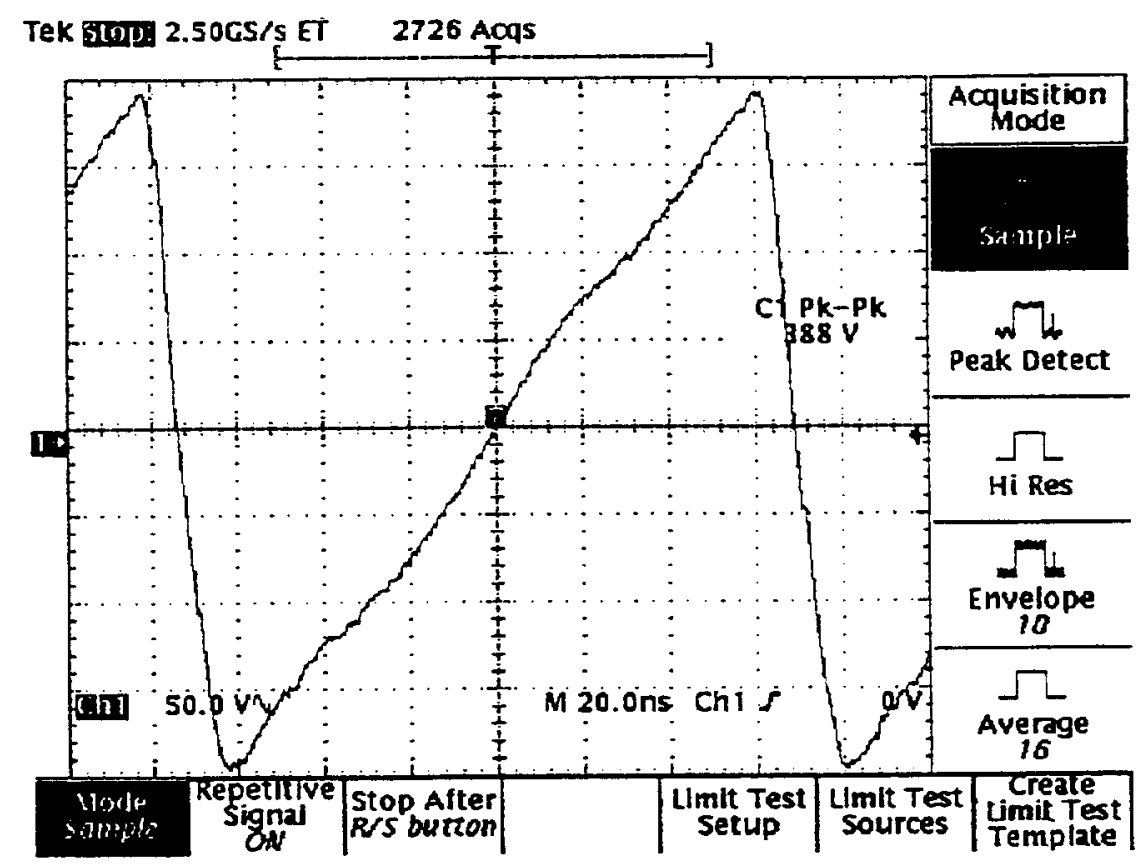

Figure 8 : the sawtooth obtained with the prototype.

5 - In beam tests of the prototype.

The buncher prototype ( sawtooth generator and buncher electrode design ) has been tested on the injection line of the GANIL C01 using a ${ }_{86} \mathrm{Kr}^{+14}$ beam produced at $\mathrm{V}_{E C R}=39674 \mathrm{~V}$, bunched and then accelerated in the C01 cyclotron at $7 \mathrm{MHz}(\beta \lambda=15.96 \mathrm{~cm})$. The transverse beam emittance was limited to the cyclotron acceptance equal to $60 \pi \mathrm{mm}$.mrad in each plane.

The usual bunching system is a double-drift harmonic buncher with drift tubes ( no grids ). To test our prototype, it was installed in between the two elements of the double drift buncher allowing a direct comparison of the results obtained using either one or the other of the two systems.

Unfortunately, there is no phase width probe in an achromatic part of the injection line in front of the cyclotron so that the comparison of the two systems is a global one, including the cyclotron effect on the beam phase and energy spreads.

The figure 9 shows the bunched beams as measured on a phase width probe located in the beam line following the cyclotron (on the extracted beam) while the figure 10 displays the turn patterns as seen on a radial probe inside the cyclotron : the difference can hardly be seen on the radial turn widths as well as on their surfaces, that means on the phase width and on the overall transmission. Moreover, the extracted beams appear to be exatly the sames in the two cases. 
Considering these first results and the simplicity of the design and of the tuning of the single gridded gap buncher, there is no doubt that it is the one we have to choose and to improve for our purpose.

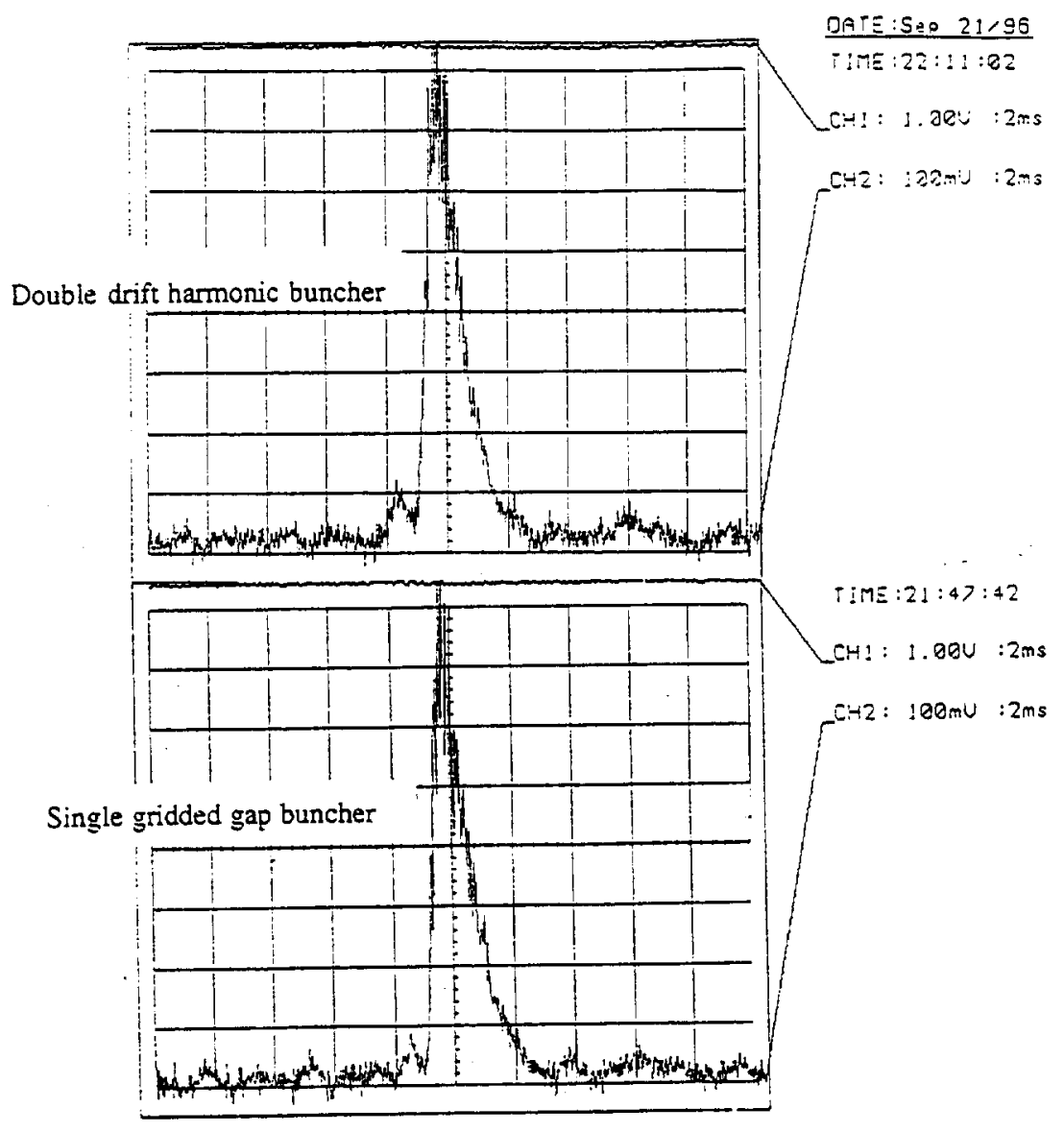

Figure $9:$ the extracted bunched beams as given by the two systems.

Double drift harmonic buncher

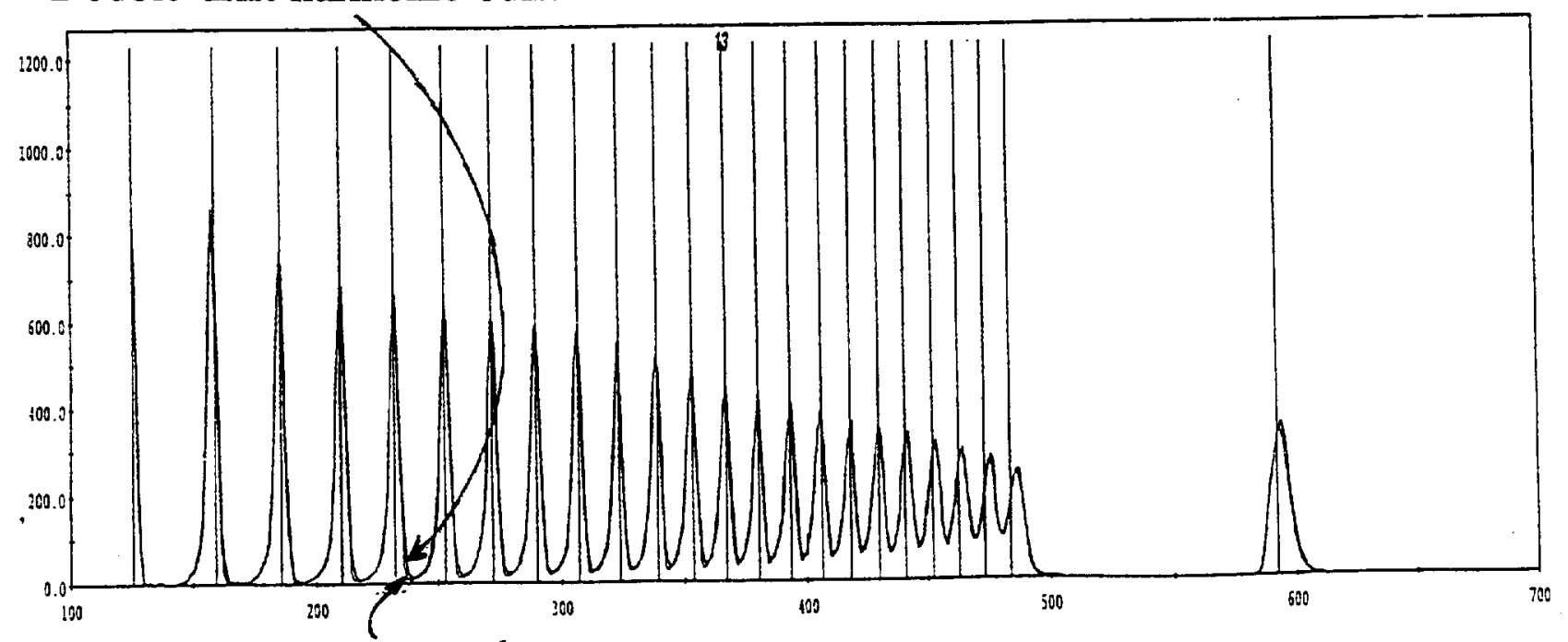

Single gridded gap buncher

Figure 10 : the turn patterns inside the cyclotron as given by the two systems. 
We also measured, the voltage being off on the buncher, the transmissions of the grids ( from a faraday cup at the object point of the injection line to a second one located before the injection into the cyclotron ) : we found a $27.5 \%$ beam loss.

The grids were built using for each one a square mesh of $30 \mu \mathrm{m}$ Mo wires obtained by photo etching. The wires, $1.5 \mathrm{~mm}$ appart, and represent $\sim 8.7 \%$ of the surface of each grid so that, due to the beam emittance and to a possible grid misalignment, we should have an $\sim 17 \%$ beam loss as compared to the $27.5 \%$ we have measured : this rather large discrepency is not well explained and a more refined study shall be performed using various wire thickness, wire spacing and trying to use a single set of wires for each grid.

\section{6 - Conclusion.}

In order to design the buncher of the radioactive ion beam facility under construction at GANIL we have decided to use a direct high amplitude saw-tooth generator as proposed at Lanzhou. We have built a prototype delivering a saw-tooth in the $\mathrm{kV}$ range between 5 and $15 \mathrm{MHz}$ which is linear within 3-4\% over more than $80 \%$ of the RF period. The dynamic of particles has been simulated in the single gridded gap system of electrodes required at our low $\beta \lambda<10 \mathrm{~cm}$, it has been shown that the stray field effects of an always positive saw-tooth can be controled by a careful design of the high voltage electrode and by adding a movable grounded plate in front which moreover allows to work at various $\beta \lambda$ with the same electrodes.

This first buncher prototype was installed and tested on the injection line of the compact injector cyclotron C01 of GANIL. The results show that our prototype is already quite efficient : it has been compared to the double drift harmonic buncher we normaly use on this line and gave the same bunching efficiency. Of course, the transmission is affected by the limited transparency of the 2 grids.

In the final design we will improve the sawtooth generator (essentialy its linearity ), the electrode design and especially the grid system which was far from optimum : some more tests are required and will be performed to improve their transparency while preserving the electric field quality, the extreme case being to put no grid! A first simulation of this 'exotic' case seems to give rather good results, the lower bunchingefficiency being more thancompensated by the gain in transparencywhen using no grid; the buncher construction and operation are much more simple ( no more problem related to the grid life time, elimination of the grid effect on the particle dynamic [3] ).This solution will also be tested on beam as soon as possible.

At higher energies the required amplitude and the frequency range of the saw-tooth would have to be increased (the choice of another tube and the lowering of the capacities should allow to get higher voltages ) and the limits of this solution have to be determined. 
Acknowledgments : we would like to thank Mrs Borniche, Ducoudret, Lechartier and Lenoble; their contributions to the design, the installation and the tests of this new bunching system were determinant

\section{References:}

[1] - 'Status of SPIRAL, the radioactive beam project at GANIL' - M.Lieuvin et al. - Proc 14th Int. Conf. Cyclotrons and their applications. Cape Town, South Africa. 1995.

[2] - 'Third harmonic simulated buncher' - C.Goldstein, A.Laisné - NIM.61 (1968) 221-225

[3] - 'Beam buncher for heavy ions' - F.Y.Linch et al. - NIM.159 (1979) 245263

[4] - 'Design of a beam buncher using a half-cosine driving function' R.C.Rogers - Proc. 13th Int . Conf. Cyclotrons and their applications.Vancouver, Canada. 1992.

[5] - Private communication. 